\title{
Musculoskeletal Conditions and Minority Ethnic Groups: Making Sense and Sensibility of Healthcare in a Global Environment
}

\author{
Ash Samanta ${ }^{1 *}$, Parthajit Das ${ }^{2}$, Ade Adebajo ${ }^{3}$, Kuntal Chakravarty ${ }^{4}$ and Mark RD Johnson ${ }^{5}$ \\ ${ }^{1}$ Consultant Rheumatologist, University Hospitals of Leicester NHS Trust, Leicester, UK \\ ${ }^{2}$ Rheumatology specialist registrar, Hospitals of Leicester NHS Trust, Leicester, UK \\ ${ }^{3}$ Professor of Rheumatology and Health Services Research, Faculty of Medicine, University of Sheffield, UK \\ ${ }^{4}$ Professor of Rheumatology, Beds and Herts Post Graduate Medical School, Queen's Hospital, Romford, UK \\ ${ }^{5}$ Professor of Diversity in Health and Social Care, Mary Seacole Research Centre, De Montfort University, UK
}

Twenty first century globalisation has been influenced by the emergence of discourse, migration patterns and the movement of ideologies across traditional national borders. In the context of healthcare, this necessitates traversing beyond the boundaries of the traditional medical model of practice. There is an urgent imperative to actively seek and include patients' perception of their illness and attitudes to medical care, in order to provide optimum personalised healthcare - one of the aspirations of current NHS policy. A challenge for globalisation is an understanding at a deeper level of the nexus between culture, health and illness. Globalisation and multiculturalism require revisiting the current delivery of health care. This editorial proposes that the key to the success of this ideal is cultural competence at the levels of individual practitioners, organisations and policymakers.

The clinical features and epidemiology of musculoskeletal disease in minority ethnic groups have been addressed relatively sparsely in the medical literature. A comprehensive review of all conditions is beyond the scope of this editorial. However, we shall emphasise key issues germane to musculoskeletal healthcare in minority populations by eliciting three exemplars: rheumatoid arthritis ("rheumatoid"), systemic lupus erythematosus ("lupus") and low serum levels of vitamin D ("hypovitaminosis D").

A relative dearth of available data makes it conspicuous as to how little research has been pursued in the UK (and elsewhere) that might influence clinical practice and policy development for quality care in subjects with rheumatoid in minority groups [1]. US studies on Afro Americans with juvenile inflammatory arthritis have reflected more severe disease with polyarticular involvement [2]. A Canadian study has shown higher serological parameters and positive family history in indigenous 'first nation' Canadians compared to the white population [3]. A study from Birmingham (UK) in South Asians has reported fewer bony erosions, higher pain and disability and similar levels of inflammation as whites [4].

It has long been recognised that migrant minorities may be subject to indirect discrimination or barriers with regard to access to healthcare [5]. A multi-centre audit of rheumatoid undertaken in four UK centres with a high minority ethnic population showed that $62 \%$ exceeded the 3 month time for referral for specialist evaluation, and there was a subsequent delay of more than 6 months in $10 \%$ of South Asian patients in initiation of disease modifying drug therapy [6]. Observations from Leicester in patients with rheumatoid suggest that South Asian subjects tend to present later with more joint deformities [2].

Appropriate educational material for diverse populations is sadly lacking [7]. It is universally recognised that hypovitaminosis D may lead to a reduction in bone mineral density (with its attendant consequences), or osteomalacia. Yet even in this eminently preventable public health condition educational material in the UK is neither fit for purpose nor culturally competent [8].
Outcome studies from Europe show a negative effect of race and ethnicity on the risk, course, morbidity and mortality of lupus and connective tissue diseases including primary vasculitides [9]. Relatively little attention has been devoted to the reasons for poorer outcome in this group. Differences in preference for treatment have been noted in minority groups in respect of rheumatoid [10]. Consequently a further question that remains to be answered is why such a differential exists?

One explanation that we propose is due to "hidden" requirements of healthcare. An understanding of prevalence, disease patterns and conventional therapeutic interventions is the orthodox medical dominant version of healthcare. Whilst in no way would we wish to decry this model, it nevertheless represents only the tip of an iceberg. What lies beneath is what we would term the "a,b,c" of understanding the poor acceptance of westernised healthcare by minority groups. This incorporates attitudes, beliefs and culture. A number of components may be identified $[1,10]$ which are as below:

a) Perception of disease: Studies suggest a generalised belief in some minority groups that rheumatoid is causally dependent upon food. "Good foods" included ginger, garlic and honey, and "bad foods" were lemon, vinegar and left over food.

b) Communication: Nuances of language and verbal expression can be an impediment to obtaining professional advice, and to confidence in the advice received.

c) Reliance on alternative therapies: An appetite exists for using alternative (non allopathic) medicine, which is perceived to be less or non-toxic.

d) Social impact: Long term conditions are perceived as being socially stigmatising, and impacting adversely on individual and family standing in society.

Global musculoskeletal healthcare needs a conceptual revision with a focus on how best it might be delivered to positively impact on minority consumers. We do not pretend that there is an easy solution.

\footnotetext{
*Corresponding author: Ash Samanta, Consultant Rheumatologist, University Hospitals of Leicester NHS Trust, Leicester, UK, E-mail: ash.samanta@uhl-tr.nhs.uk

Received November 19, 2013; Accepted November 20, 2013; Published November 25, 2013

Citation: Samanta A, Das P, Adebajo A, Chakravarty K, Johnson MRD (2013) Musculoskeletal Conditions and Minority Ethnic Groups: Making Sense and Sensibility of Healthcare in a Global Environment. J Gen Pract 1: e104. doi: 10.4172/2329-9126.1000e104

Copyright: (c) 2013 Samanta A, et al. This is an open-access article distributed under the terms of the Creative Commons Attribution License, which permits unrestricted use, distribution, and reproduction in any medium, provided the original author and source are credited.
} 
Citation: Samanta A, Das P, Adebajo A, Chakravarty K, Johnson MRD (2013) Musculoskeletal Conditions and Minority Ethnic Groups: Making Sense and Sensibility of Healthcare in a Global Environment. J Gen Pract 1: e104. doi: 10.4172/2329-9126.1000e104

Page 2 of 2

What we propose is a framework for advancing the agenda in this area, in the hope that it may have universal applicability in a "global village" that aspires towards cultural integration. Over the last few years our small working party of committed professionals in the UK has tasked itself with highlighting and promoting awareness of these particular issues through regular national and regional meetings of the special interest group (in ethnicity) of the BSR and in Leicester, through multidisciplinary engagement of academics, practitioners and patient and public.

Our proposed consensus framework for global application in the area of ethnic minority musculoskeletal health is constructed upon the following tenets: actively listening to the views of minority groups; knowledge management of cultural differences through collaboration between healthcare researchers; providing experiential approaches to holistic care; integrating models of best practice across disease-specific boundaries; promoting policy and organisational development for culturally competent services; prioritising unmet needs of minority groups at the level of macro-economics for healthcare; and developing an international database accessible to healthcare providers, professionals and users.

Universality can only be achieved through a genuine engagement with diversity. This is particularly true with the health of the world - a host country must open its heart to diversity. As expressed pithily by Ruskin in his autobiography regarding his experience in Siena: "Cor magis tibi Sena pandit" ["More than her gates, Siena opens her heart to you"].

\section{Disclosure Statement}

Ash Samanta: Former Chair Leicester Centre for Ethnic Research; Organiser, Leicester Conference on Ethnicity and Rheumatology 2011 \& 12 for which pharmaceutical sponsorship and speaker fees were received; Received Pfizer Foundation and co-recipient arc funding for work on educational materials for RA and osteomalacia, some results of which are contained in this editorial.

\section{References}

1. Samanta A, Samanta J, Johnson MRD (2005) Rheumatoid arthritis in minority ethnic groups: patterns of disease, clinical and sociocultural features among British South Asians. Diversity in Health and Social Care 2: 99-118.

2. Graham TB, Glass DN (1997) Juvenile rheumatoid arthritis: ethnic differences in diagnostic types. J Rheumatol 24: 1677-1679.

3. Jacono J, Jacono B, Cano P, Segami M, Rubin L (1996) An epidemiological study of rheumatoid arthritis in a northern Ontario clinical practice: the role of ethnicity. J Adv Nurs 24: 31-35.

4. Griffiths B, Situnayake RD, Clark B, Tennant A, Salmon M, et al. (2000) Racial origin and its effect on disease expression and HLA-DRB1 types in patients with rheumatoid arthritis: a matched cross-sectional study. Rheumatology (Oxford) 39: 857-864.

5. Johnson M (2003) Ethnic Diversity in Social Context. In: Kai J (Ed.), Ethnicity, Health and Primary Care. Oxford: Oxford University Press pp. 3-13.

6. Samanta A, Shaffu S, Panchal S, Morthy A, Hassan W, et al. (2012) Tinkering at the edges or collaborative symbiosis? Ethnicity and rheumatology: a consensual review discussion. Diversity and Equality in Health and Care 9: 209-217.

7. Adebajo A, Blenkiron L, Dieppe $P$ (2004) Patient education for diverse populations. Rheumatology (Oxford) 43: 1321-1322.

8. Samanta A, Johnson MR, Guo F, Adebajo A (2009) Snails in bottles and language cuckoos: an evaluation of patient information resources for South Asians with osteomalacia. Rheumatology (Oxford) 48: 299-303.

9. Piram M, Maldini C, Mahr A (2012) Effect of race/ethnicity on risk, presentation and course of connective tissue diseases and primary systemic vasculitides. Curr Opin Rheumatol 24: 193-200.

10. Kumar K, Gordon C, Toescu V, Buckley CD, Horne R, et al. (2008) Beliefs about medicines in patients with rheumatoid arthritis and systemic lupus erythematosus: a comparison between patients of South Asian and White British origin. Rheumatology (Oxford) 47: 690-697. 